\title{
A numerical method for computing unstable quasi-periodic solutions for the 2-D Poiseuille flow ${ }^{\dagger}$
}

\author{
Pablo S. Casas ${ }^{1}$ and Àngel Jorba ${ }^{2}$ \\ ${ }^{1}$ Departamento de Matemática Aplicada I. Universidad Politécnica de Cataluña. \\ Diagonal, 647; 08028 Barcelona. Spain. e-mail: pablo@vilma.upc.es \\ ${ }^{2}$ Departamento de Matemática Aplicada y Análisis. Universidad de Barcelona. \\ Gran Via, 585. 08007 Barcelona. Spain. e-mail: angel@maia.ub.es
}

We study the dynamics of two-dimensional Poiseuille flow. Firstly we obtain the family of periodic solutions which bifurcates from the laminar flow, together with its stability for several values of the wave number $\alpha$. The curve of periodic flows presents several Hopf bifurcations. For $\alpha=1.02056$ we follow the branches of quasi-periodic orbits that are born at one of the bifurcation points.

\section{Poiseuille flow}

We consider the flow of a viscous incompressible fluid, in a channel between two parallel walls at $y= \pm 1$, driven by a constant stream-wise pressure gradient and governed by the dimensionless Navier-Stokes equations in primitive variable formulation

$$
\frac{\partial \mathbf{u}}{\partial t}+(\mathbf{u} \cdot \nabla) \mathbf{u}=-\nabla p+\frac{1}{R e} \Delta \mathbf{u}
$$

where $\mathbf{u}=(u, v)$ are the components of the velocity, $p$ is the pressure and $R e$ is the Reynolds number defined in terms of the constant pressure gradient. As boundary conditions we suppose $u(x, \pm 1, t)=v(x, \pm 1, t)=0$ and $(u, v, p)(x+L, y, t)=(u, v, p)(x, y, t)$ for $L=2 \pi / \alpha, x \in$ $\mathbb{R}, y \in[-1,1], t \geq 0$. The initial profile of velocities is simply subjected to the incompressibility condition, $\operatorname{div} \mathbf{u}=0$. We are concerned with the dynamics of Poiseuille flow in varying the parameters $R e$ and $\alpha$. The stability of the laminar solution has been studied extensively through the literature. For instance, Orszag [1] obtained the critical Reynolds number, $R e_{\mathrm{cr}}=$ 5772.22 for $\alpha=1.02056$, so that if $R e<R e_{\mathrm{cr}}$ the laminar solution is stable for any value of $\alpha$.

We construct a numerical integrator of system (1) by means of a spectral method. Since $u, v, p$ are supposed periodic in $x$, we replace them by their truncated Fourier series:

$$
(u, v, p)(x, y, t)=\sum_{k=-N}^{N}\left(\hat{u}_{k}, \hat{v}_{k}, \hat{p}_{k}\right)(y, t) e^{i k \alpha x}, \quad x \in \mathbb{R}, \quad y \in[-1,1], \quad t \geq 0 .
$$

We resolve the Fourier coefficients evaluating them at the Chebyshev abscissas. Next we eliminate $v$ and $p$ from the discretized system to obtain a set of ODE just in terms of $u, \partial u / \partial t=$ $f(u)$. This system has dimension $(2 N+1)(M-2)+1$, being $M$ the number of Chebyshev modes in $y$. For the temporal discretization we employ a semi-implicit finite difference method: Adams-Bashforth for advection and Crank-Nicolson for pressure and viscosity terms.

\section{Periodic solutions}

Due to the translational symmetry of the channel in $x$, it is showed in [3] that any timeperiodic function $w(x, y, t)$, of period $T$, is a rotating wave, i.e. $w(x, y, t)=w(x-c t, y, 0)$, for $c=L / T$. In this way we may find periodic solutions in time as stationary flows in a system

$\dagger$ Research partially supported by funds from the Dept. Matemática Aplicada I (Universitat Politècnica de Catalunya), the Spanish grant DGICYT PB94-0215 and the Catalan grant CIRIT 1998S0GR-00042. 
of reference moving with velocity $c$ in the stream-wise direction, which can either correspond to stable or unstable time-periodic orbits. Given a profile of velocities $(u, v)$ we define its amplitude $A$, as the distance to the laminar solution $\overline{\mathbf{u}}=(\bar{u}, 0), \bar{u}=1-y^{2}$, in the $L_{2}-$ norm.

The bifurcating diagram for the periodic flows in the $R e-A$ plane, together with their stability is represented in figure 1 . We decide the stability of a time-periodic solution $u$ upon the eigenvalues of the Jacobian matrix $D f(u)$. In the case of the laminar flow, we obtain the classical results of Orszag [1] about the critical Reynolds being at $R e_{\mathrm{cr}}=$ 5772.22 for $\alpha=1.02056$. First, the bifurcation curve of periodic flows reaches the laminar solution at $R e_{\mathrm{cr}}$ and in addition, the laminar solution

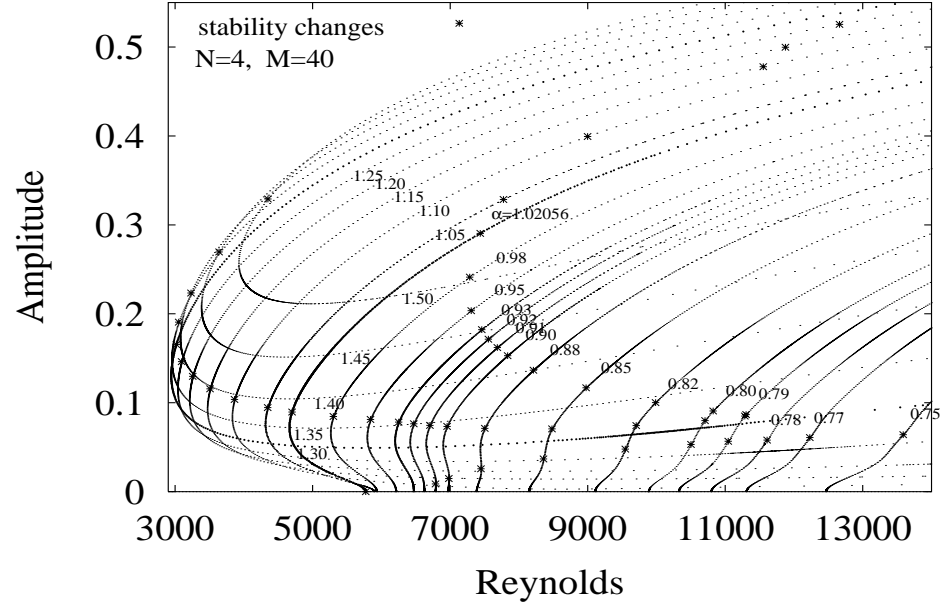

Fig. 1. Bifurcating curves for several values of $\alpha$. is checked to be stable when $R e<R e_{\mathrm{cr}}$ and unstable if $R e>R e_{\mathrm{cr}}$. Soibelman \& Meiron [4] found analogous bifurcations for $\alpha=1.1$, and the critical Reynolds number for which there are time-periodic solutions: $R e \approx 2900$ for $\alpha \approx 1.3$. In figure 1 this minimum Reynolds number is verified. We also notice that there exists an attractor periodic solution for $R e$ as low as 3000 .

\section{Quasi-periodic solutions}

In this section we fix $\alpha=1.02056$. Taking $R e \approx 7500$, the corresponding periodic flow is unstable so, as a test, we use it as initial condition. After some steps of numerical integration, the solution seems to fall in a regular régime. Then, in figure 2 , we plot the projection of the solution vector $u(t)=\left(u_{1}, \ldots, u_{K}\right)(t), K=(2 N+1)(M-2)+1$, over the plane of two arbitrary coordinates (the $120^{\text {th }}$ and $232^{\text {th }}$ to be precise). Each point in figure 2(a) corresponds to the value of the specified coordinates at a time instant. As we can observe, the trajectory seems to fill densely the projection of a torus. Taking an ad hoc Poincaré section $\Sigma$, of the space of solutions $u(t)$ and plotting the same coordinates as above, but only when crossing the Poincaré section, we see in figure 2(b), a closed curve, which confirms us that our flow lives in a 2-torus.

In [3] it is proved that, with the translational symmetry of the channel, every solution $w(x, y, t)$ that lies on an isolated invariant 2-torus, not asymptotic to a rotating wave, is a modulated wave, that is, exists $\tau>0$ and $\phi \in \mathbb{R}$ such that for every integer $n$ we have $w(x, y, n \tau)=w(x+n \phi, y, 0)$. As a consequence, this kind of wave has the property that, may be viewed as a periodic wave of period $\tau$ in a frame of reference moving with speed $c=(p L-\phi) / \tau$, for any integer $p$, as is easily proved. For each value of $c$ we construct a Poincaré map as above $P_{c}: \Sigma \longrightarrow \Sigma$. Starting from an initial condition $u=u(0), u \in \Sigma$ we integrate (1) for an observer that moves with speed $c$, until a time $\tau$ such that $u(\tau) \in \Sigma$ and crosses it in the same sense as it did initially. We set $P_{c}(u)=u(\tau)$ and we try to find $u, c$ such that $P_{c}(u)=u$. With this $c$ the quasi-periodic flow $u$ will be viewed as a periodic one.

To find the branches of quasi-periodic solutions at the bifurcation point $R e \approx 7400$, we search for zeros of the map $F(c, u)=P_{c}(u)-u$, using as a starting guess, points on a mesh around the bifurcating periodic solution $u^{*}$. This mesh is selected from the directions in the 
kernel of $D F\left(u^{*}\right)$. Taking $R e$ as a continuation parameter, we can trace a curve of quasi-periodic flows in the $R e-A$ plane as above. We emphasize that zeros of $F(c, u)$ can either correspond to stable or unstable quasi-periodic solutions. Unlike the periodic flow, in this case the amplitude $A$ of the solution does depend on time so we evaluate it when $u \in \Sigma$. As we observe in figure 3(a), there is just one branch of quasi-periodic solutions which bifurcates from the curve of periodic flows. If we plot, instead of the amplitude, the value of a selected coordinate of the solution vector, we obtain the graph in figure 3(b), which confirms us the existence of two different branches of quasi-periodic orbits on the Hopf bifurcation at $R e \approx 7400$. As the periodic orbits go from stable to unstable when crossing the bifurcation point, the two branches of quasi-periodic solutions are locally stable to two-dimensional disturbances. By means of the eigenvalues of the Jacobian matrix $\partial P_{c} / \partial u(u)$ we can also estimate the stability of the obtained quasi-periodic flows. The continuation of those bifurcating branches, their changes of stability and other bifurcation curves is actually a work in progress.
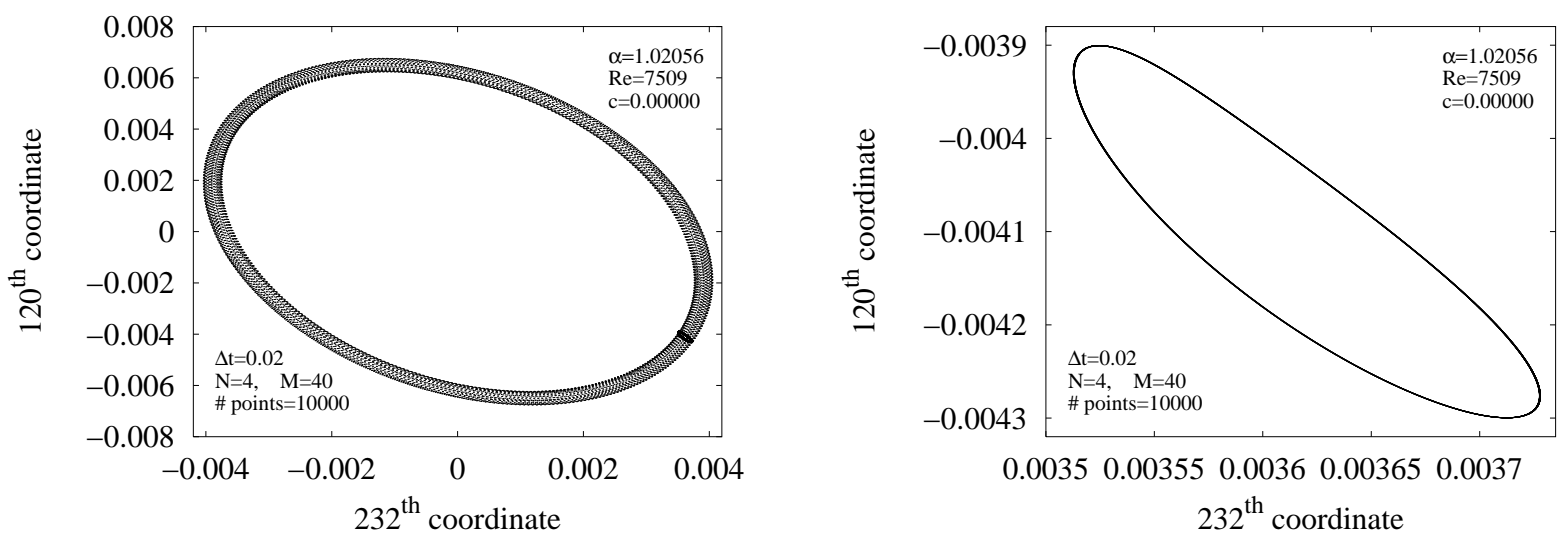

Fig. 2. Solution vector (a) each $\Delta t$ units of time and (b) on the Poincaré section.
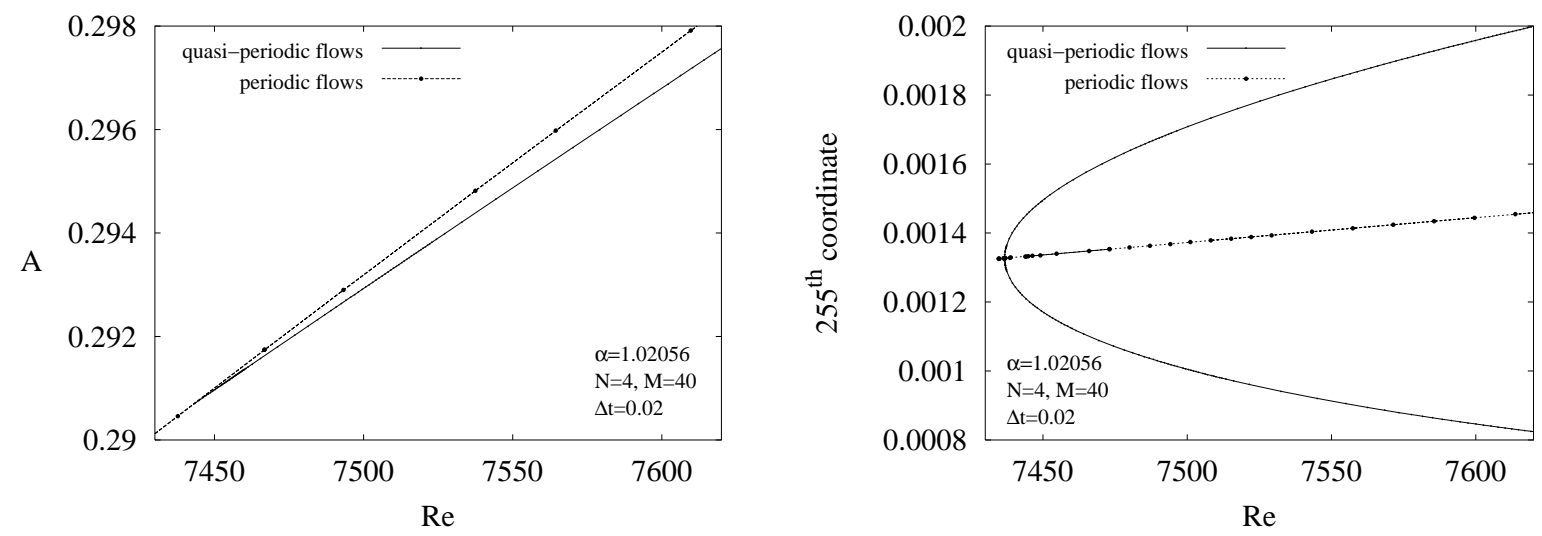

Fig. 3. Bifurcating curves of quasi-periodic flows.

\section{References}

[1] S.A. Orszag. Accurate solution of the Orr-Sommerfeld stability equation. J. Fluid Mech., 50-4:689-703, 1971.

[2] J.D. Pugh and P.G. Saffman. Two-dimensional superharmonic stability of finite-amplitude waves in plane Poiseuille flow. J. Fluid Mech., 194:295-307, 1988.

[3] D. Rand. Dynamics and symmetry predictions for modulated waves in rotating fluids. Arch. Rat. Mech. Anal., 79:1-37, 1982.

[4] I. Soibelman and D.I. Meiron. Finite-amplitude bifurcations in plane Poiseuille flow: two-dimensional Hopf bifurcation. J. Fluid Mech., 229:389-416, 1991. 